\title{
Telemedicina e uberização da saúde: médicos operários ou consumidores?
}

Telemedicine and uberization of health: doctors as workers or consumers?

Telemedicina y uberización de la salud: ¿médicos como trabajadores o consumidores?

\author{
José Augusto Fontoura Costa ${ }^{1}$ \\ Fernanda Sola ${ }^{2}$ \\ Marco Aurélio Fernandes Garcia ${ }^{3}$
}

\begin{abstract}
Resumo
Objetivo: o artigo visa analisar as mudanças na categorização legal dos médicos devido a possíveis mudanças na profissão médica provocadas pela telemedicina, vislumbrando-se uma possível precarização das relações de trabalho médicas e a uberização da medicina. Metodologia: a abordagem metodológica parte dos conceitos de custos de transação e de informação desenvolvidos pela economia neoinstitucional e da análise sociológica jurídica, feitas para definir um cenário possível da profissão médica e de sua estrutura legal. $O$ tipo ideal de telemedicina mediada por uma plataforma digital dominante é o ponto de partida para a análise legal de possíveis categorizações de médicos e serviços de saúde. Com base nesse modelo ideal, são traçadas diversas considerações sobre as suas possíveis consequências para o exercício da profissão médica. Resultados: a principal contribuição é a discussão legal da rivalidade entre a categorização como trabalhador ou consumidor, bem como uma possível dupla incidência de regimes legais. Conclusão: os papéis futuros dos médicos como profissionais, trabalhadores ou consumidores dependerão não apenas da dinâmica do mercado, mas centralmente do quadro jurídico. O controle da qualidade e dos benefícios sociais dos serviços de saúde depende do arcabouço legal, na medida em que estão vinculados aos papéis dos profissionais.
\end{abstract}

Palavras-chave: Telemedicina. Políticas de eSaúde. Defesa do consumidor. Categorias de trabalhadores.

\begin{abstract}
Objectives: the article aims to analyze changes in the legal categorization of doctors due to possible changes in the medical profession caused by telemedicine. It envisions a possible precariousness of medical work relationships and the uberization of medicine. Methods: the methodological approach starts from the concepts of transaction costs and information developed by the neoinstitutional economy and from the sociological legal analysis made to define a possible scenario of the medical profession and its legal structure. The ideal type of telemedicine mediated by a dominant digital platform is the starting point for the legal analysis

\footnotetext{
${ }^{1}$ Doutor e livre docente; professor da Pós-graduação em Direito, Universidade Ceuma. São Luís, Maranhão, Brasil. https://orcid.org/0000-0003-2300-3258.E-mail: jafontouracosta@gmail.com

2 Doutora; professora da Pós-graduação em Direito, Universidade Ceuma, São Luís, Maranhão, Brasil. https://orcid.org/0000-0001-7926-6378. E-mail: sollafernanda@gmail.com

3 Mestre, Universidade de São Paulo, São Paulo, SP, Brasil. https://orcid.org/0000-0002-5717-3792. E-mail: marcofg0@hotmail.com
} 
of possible categorization of doctors and health services. Based on this ideal model, several considerations are outlined about its possible consequences for the exercise of the medical profession. Results: the main contribution is the legal discussion of the rivalry between categorization as worker or consumer, as well as a possible double incidence of legal regimes. Conclusions: the future roles of doctors as professionals, workers, or consumers will depend not only on the dynamics of the market, but centrally on the legal framework. The control of the quality and social benefits of health services depends on the legal framework, insofar as they are linked to the roles of professionals.

Keywords: Telemedicine. eHealth policies. Consumer advocacy. Occupational groups.

\section{Resumén}

Objetivos: el artículo tiene como objetivo analizar los cambios en la categorización jurídica de los médicos por posibles cambios en la profesión médica provocados por la telemedicina, vislumbrando una posible precariedad de las relaciones laborales médicas y la uberización de la medicina. Metodología: el abordaje metodológico parte de los conceptos de costos de transacción e información desarrollados por la economía neoinstitucional y del análisis jurídico sociológico, realizado para definir un posible escenario de la profesión médica y su estructura jurídica. El tipo ideal de telemedicina mediada por una plataforma digital dominante es el punto de partida para el análisis legal de posibles categorizaciones de médicos y servicios de salud. A partir de este modelo ideal, se esbozan varias consideraciones sobre sus posibles consecuencias para el ejercicio de la profesión médica. Resultados: el principal aporte es la discusión jurídica de la rivalidad entre categorización como trabajador o consumidor, así como una posible doble incidencia de regímenes legales. Conclusiones: los roles futuros de los médicos como profesionales, trabajadores o consumidores dependerán no solo de la dinámica del mercado, sino centralmente del marco legal. El control de la calidad y los beneficios sociales de los servicios de salud depende del marco legal, en la medida en que están vinculados a los roles de los profesionales.

Palabras clave: Telemedicina. Políticas de eSalud. Defensa del consumidor. Grupos profesionales.

\section{Introdução}

Enquanto nos Estados Unidos há plataformas que congregam milhares de médicos e pacientes, a telemedicina brasileira apenas ensaia seus primeiros passos. Aqui, por enquanto, a oferta é materializada por seguradoras e hospitais, interessados no barateamento dos serviços e na expansão do alcance de sua atuação, respectivamente. Identifica-se, no presente, um modesto movimento para a consolidação de plataformas de prestação de serviços médicos por atendimento presencial e remoto, com precificação e critérios de escolha diferenciados, o que provavelmente associa-se à falta de segurança regulatória (1).

A possibilidade técnica de separação de etapas produtivas e as vantagens econômicas da especialização apontam para um mercado onde empresas especializadas 
sejam incumbidas de administrar os suportes tecnológicos e os relacionamentos com os profissionais: as empresas provedoras de telemedicina.

O presente artigo tem a finalidade de discutir a potencial realocação da posição dos médicos em face de plataformas de intermediação por meio de uma abordagem interdisciplinar entre Direito e Economia. Para tanto, se realiza uma análise com fundamento em pressupostos da economia neoinstitucional - sobretudo a presença de custos de informação e de transação - para compreender o modelo de oferta de serviços e atenção médica. A partir dos modelos de estrutura e fluxo, busca-se verificar a posição jurídica que os médicos poderiam vir a assumir em um novo modelo de consultas remotas sincrônicas possibilitado pela telemedicina. A temática da regulação da telemedicina, envolvendo quais instituições seriam legitimadas para tanto e qual seria o conteúdo material da regulação, não são objeto do presente artigo.

No contexto da crescente implementação de serviços de telemedicina, compreendidos como "exercício da medicina mediado por tecnologias para fins de assistência, pesquisa, prevenção de doenças e lesões e promoção de saúde" (2), é possível identificar transformações tendentes a implicar a redefinição econômica e social dos papéis de médicos, hospitais e seguros de saúde, assim como das emergentes prestadoras de telemedicina.

Por isso, a primeira parte deste artigo propõe uma estrutura analítica da conformação dos vínculos entre os atores relevantes. Destaca-se a uberização de parcela dos serviços médicos decorrente da deslocalização da prestação, da homogeneização dos serviços, da administração de excedente de trabalho mediante vínculos precários e do controle do manejo institucional local pelas empresas de intermediação.

Observe-se a intencional ausência da avaliação do papel do Estado como provedor e financiador de serviços de saúde, fundamental na sistemática brasileira constitucionalmente alicerçada. Justifica-se tal opção por duas razões: i) simplificar o modelo de análise, considerando todos os atores como presentes em um mercado e orientados por motivações exclusivamente econômicas, já que a inclusão da ação estatal elevaria a complexidade e forçaria a construção de alguns cenários alternativos e ii) o objetivo de restringir a análise jurídica à roupagem jurídica da posição contratual dos médicos em face das plataformas de intermediação. 
A hipótese central é de que o médico pode passar a assumir a condição jurídica de consumidor dos serviços de intermediação oferecidos pelas plataformas digitais, sem assumir a posição de trabalhador empregado. Não obstante, tal circunstância não serve à recomposição da condição de profissional liberal predominante entre o século XIX e as décadas de 60 e 70 do século XX (3), mas a uma precarização das condições de trabalho.

É importante destacar o fato de não se buscar, no âmbito deste artigo, compreender as eventuais modificações da estrutura administrativa e regulatória do exercício da medicina e da oferta de serviços associados. Embora esses aspectos sejam de fundamental importância e exista uma evidente relação de causalidade bilateral entre as estruturas contratuais e negociais, por um lado, e as formas regulatórias efetivas, pelo outro, não faz sentido buscar abarcar todos os aspectos em um artigo.

\section{A estrutura básica de serviços de telemedicina}

Cerca de dez anos atrás, o relatório da Organização Mundial da Saúde sobre a telemedicina já apontava a existência de mais de uma centena de definições, antes de adotar um conceito bastante amplo, considerando a telemedicina como a prestação de serviços médicos a distância com a utilização de tecnologias da informação sincrônicas. Para os fins deste trabalho, adota-se uma definição mais restrita: a prestação de serviços de consulta e atenção médica mediante tecnologias de informação sincrônicas e capazes de mimetizar a presença médica, como videoconferência (4). Esses serviços podem ser prestados pelo médico em duas modalidades básicas: atendimento médico-médico (orientação e/ou pesquisa) e médico-paciente (consulta), com ou sem a presença de intermediadores e seguradoras. O objeto do presente artigo, porém, abrange apenas a segunda modalidade. Ademais, reconhece-se a existência de limites inerentes à prestação de serviços de telemedicina, em particular a impossibilidade de realização de exame físico durante a consulta. Não se trata, por óbvio, de delineamento com pretensões de verdade, mas de mera definição de trabalho.

Em termos metodológicos, as previsões sobre possíveis cenários para a avaliação da telemedicina comercial procuram compreender a reestruturação do mercado de serviços médicos na forma dita uberizada. Isso se refere aos efeitos profundos no mercado do uso de plataformas de intermediação entre consumidores e prestadores individualizados de serviços por meio da extrapolação para o mercado privado de serviços de saúde de 
observações teóricas gerais (5-7) e estudos de caso em outros mercados (8-10). A literatura consolidada de economia neoinstitucional permite ressaltar o papel da construção jurídica dos elementos básicos das trocas de mercado e da estruturação administrativa e da relação entre os custos de agência e custos de transação (11-13), de modo possibilitar a discussão do quadro regulatório e, por certo, de sua fragilidade atual (1).

Formas diversas de telemedicina existem desde o momento em que essas tecnologias de informação sincrônicas foram desenvolvidas. Recomendações para condução de telemedicina de forma responsável e ética foram formuladas ainda em 1999 pela Associação Médica Mundial (14). No Brasil, programas governamentais (como, por exemplo, o Programa Nacional de Telessaúde) e a iniciativa privada (por exemplo, o uso de telemedicina para fins exclusivos de pesquisa em hospitais) já representavam o uso crescente da telemedicina não-comercial (15)). A discussão deste artigo é centrada na modalidade de telemedicina comercial, particularmente em relação à modalidade médicopaciente, recentemente aprovada no país.

A telemedicina comercial, sob a perspectiva dos custos aplicáveis ao provedor de serviços, tipicamente inclui custos relacionados ao uso sistema de comunicação sincrônica, sistemas de monitoramento remotos (se aplicáveis), telefones, e-mails, call centers (se aplicáveis), entre outros, assim como envolve a remuneração da hora colocada à disposição pelo médico. Contudo, a telemedicina não envolve, necessariamente, a disponibilização do espaço hospitalar para a realização do serviço de atenção médica (16).

Porém, e nesse aspecto se concentra este artigo, os custos da telemedicina comercial podem ser vistos da perspectiva do médico. Nesse caso o médico recebe o serviço de intermediação, o qual envolve a captação do paciente e sua alocação aos médicos usuários do sistema. Ativos imateriais, como o uso da marca e os efeitos de rede, evidenciam-se desse ponto de vista, já que a diferença entre boas e más plataformas dependerá da capacidade de atrair os pacientes por meio de sua reputação e imagem projetada.

Não há uma particular limitação de ramos de medicina que possam ser objetos de telemedicina, embora determinadas áreas sejam mais apropriadas para consultas a distância do que outras, como fica evidente, por exemplo, quando comparamos a atenção médica pré-natal e cuidados típicos das unidades de terapia intensiva.

Os efeitos econômicos imediatamente previsíveis para a adoção da telemedicina seriam associados à troca de custos de localização, como aluguéis, estacionamentos, 
estrutura de acolhimento e deslocamentos de médicos para os locais de trabalho, pelos custos dos serviços de intermediação. Observe-se, a propósito, que os primeiros envolvem vários elementos que compõem investimento em capital fixo, enquanto o outro, do ponto de vista do médico, representa predominantemente custos marginais, já que as contribuições mais significativas se dão on demand, ou seja, por consulta efetivamente realizada.

Há, nesse sentido, várias vantagens do modelo a distância, entre os quais a redução dos custos, a facilidade de ingresso de novos ofertantes, o aumento da extensão geográfica do mercado e a padronização dos serviços, gerando competição por preços e pressão para aumento da eficiência. Interessante observar, nesse sentido, a possível sobreposição de três sistemas de controle de qualidade e adequação da oferta: i) o das autoridades públicas competentes, como Ministério e Secretarias da Saúde; ii) o das associações profissionais, como o Conselho Federal de Medicina e a Associação Médica Brasileira e iii) o do mercado, já que a reputação e confiança passam a ser ativos imateriais de imensa importância para as empresas de intermediação, que buscariam manter a qualidade dos serviços médicos em patamares que não comprometam sua capacidade de amealhar clientes.

\section{A estrutura produtiva da telemedicina e seus efeitos sobre a oferta de consultas médicas}

A oferta de atenção médica por telemedicina é mais barata que o modo presencial tradicional. Embora incorpore custos de implementação da tecnologia e remunere o fluxo informacional, há redução dos custos de instalação da clínica médica e de deslocamento dos pacientes. A primeira resulta da possibilidade de localizar fisicamente o médico em regiões onde imóveis são mais baratos, de dispensar espaços para receber pacientes e de dispensar funcionários de secretaria e recepção. A segunda decorre da possibilidade de o paciente acessar o médico em seu telefone móvel ou de um terminal doméstico de computador, sem ter de utilizar o tempo e os meios necessários para o deslocamento, às vezes até mesmo penoso.

Tanto na medicina tradicional, quanto na telemedicina comercial, há uma remuneração pelo fluxo informacional unidirecional médico-paciente. Contudo, na telemedicina não há a incorporação de uma pletora de custos fixos para a prestação do serviço, incorridos e suportados, em regra, pela plataforma de intermediação. 
Em termos econômicos há alterações do lado da oferta e do lado da procura. Pela oferta, há evidente redução dos custos fixos de oferta, facilitando o ingresso de novos fornecedores. Do mesmo modo, há redução dos custos marginais, o que altera a relação de oferta para um patamar superior. Fossem essas as únicas alterações, se esperaria uma redução dos preços acompanhada por um aumento da quantidade de consultas efetivamente contratadas.

Em princípio, a procura também pode vir a experimentar um incremento, pois os obstáculos para chegar até os centros de atendimento seriam superados. Para algumas especialidades para as quais as dificuldades de deslocamento sejam mais significativas, como a geriatria, poder-se-ia esperar um maior impacto do que em outras. Os efeitos de um aumento da demanda, coeteris paribus, é o incremento tanto dos preços, quanto das quantidades efetivamente adquiridas. Como, porém, há aumento concomitante da oferta (em razão da redução dos custos marginais) e da procura (pelo aumento dos custos de oportunidade) é possível prever o incremento da quantidade de consultas, embora não se possa prever com segurança o efeito sobre os preços. A literatura aponta para a redução de custos, mas há poucos estudos empíricos a respeito (17) e as evidências empíricas são apenas moderadamente animadoras quanto aos benefícios (18), embora as perspectivas sejam bastante boas se houver a estruturação adequada (19).

Porém, esse é um mercado em que a imensa assimetria de informação e presença de problema de agência entre paciente e médico implicam uma situação bem longe da perfeição. Além disso, a vulnerabilidade decorrente do estado de saúde e a incomensurabilidade da vida e do bem-estar tornam o cálculo econômico racional improvável, se não impossível. Não obstante, a presença das empresas de seguro e planos de saúde, bem como a presença de oferta estatal, geram efeitos sobre a precificação, já que os médicos passam a ajustar os valores com intermediários e não com consumidores finais (20).

Em princípio as plataformas parecem vir a ocupar uma posição que se assemelha e compete com planos de saúde e hospitais pelos benefícios econômicos. O médico também tem uma posição relativamente vulnerável em face destes sistemas, inclusive porque não compartilha com os gestores das plataformas a capacidade econômica e a expertise para administrar não apenas sistemas informáticos, mas estruturas sofisticadas de potencialização das redes para dominar mercados. É nesse ponto que o profissional passa 
a compartilhar uma vulnerabilidade com seus pacientes: a assimetria de informação em relação a seus fornecedores.

Não obstante, há outro conjunto de potenciais efeitos com grande relevância para a estruturação da produção. Pelo menos para a oferta de consultas e atenção médica que dispensa a presença efetiva, contentando-se com o mimetismo, devem ser esperadas modificações profundas no modo de relacionamento entre pacientes e prestadores de serviços, bem como nos papéis dos atores envolvidos.

Com efeito, uma consulta médica normal de hoje ou é marcada diretamente pelo paciente com o médico de sua escolha ou, sobretudo em situações de emergência, seguindo a regra conforme a qual, respeitadas eventuais prioridades, o primeiro a chegar é atendido antes nas filas de hospitais. Certas plataformas digitais permitem o agendamento de consultas diretamente com os profissionais médicos, que atendem os pacientes em sua própria clínica. Podemos apontar os seguintes exemplos destas plataformas: BoaConsulta 4 , Doctoralia $^{5}$ e Doctena 6 . O financiamento por meio de seguro de saúde pode limitar o rol de médicos à rede conveniada, sem alterar profundamente o modelo de escolha. Não obstante, a presença física do médico sempre foi uma constante na atenção médica comercial. Separado o paciente do médico, mas sendo mantido o fluxo informacional que caracteriza a atenção médica, há um natural descolamento da medicina do locus que ela era usualmente realizada - o hospital.

\section{Medicina em rede: confiança e instâncias legitimadoras na telemedicina}

A escolha da medicina como instrumento para cuidar da saúde e a especifica identificação dos hospitais, médicos e especialidades médicas relevantes passam por processos sociais de construção de legitimidade e confiança bastante específicos e dependentes dos modelos institucionais e regulatórios existentes. As próprias escolhas dos pacientes são naturalmente orientadas pelas instâncias legitimadoras.

Em primeiro lugar, quando a medicina é escolhida em detrimento de formas tradicionais, religiosas e alternativas, é inegável o papel legitimador geral das associações profissionais (conselhos regionais de medicina, por exemplo) $(21,22)$, lugares de formação

\footnotetext{
${ }^{4}$ https://www.boaconsulta.com

${ }^{5}$ https://www.doctoralia.com.br

${ }^{6}$ https://pt.doctena.lu
} 
(faculdades e hospitais) e lugares de prática (hospitais) (23). Além disso, as redes locais de confiança e reputação são importantes determinantes da escolha específica de um profissional e não de outro.

Nas últimas décadas, porém, o modelo de legitimação centrado na atuação das associações profissionais vem, inclusive por bons motivos, sofrendo uma contínua erosão (24). A justificativa generalizada de uma complexidade suficientemente grande para evitar a ingerência de pessoas externas às corporações para realizar a seleção dos ingressantes e o controle ético e técnico do exercício foi perdendo sustentação na medida em que as entidades estatais responsáveis pela oferta ou regulação dos serviços de saúde passaram a atuar mais e os seguros, planos de saúde e hospitais privados passaram a controlar os custos e a precificação de serviços e equipamentos. Para empregar as categorias propostas por Eliot Freidson (25), pode-se dizer que a lógica do profissionalismo vem sendo suplantada pelas lógicas da burocracia estatal e do mercado.

Boa parte da perda da centralidade das associações pode ser relacionada à já crescente parcela de trabalho assalariado em substituição à atividade médica como profissão liberal, fenômeno ora associado à lógica dos mercados, ocasionando a proletarização $(26,27)$, ora à predominância da contratação pública (28-30). Em termos de efeitos sobre a esfera do trabalho, a uberização é diversa da proletarização, pois se afasta das percepções correntes sobre trabalho e emprego para admitir estruturas bem mais flexíveis.

Resta, evidentemente, a disputa pelo quadro jurídico e institucional, posto que o profissionalismo moderno não se esgota na dinâmica social empoderadora das associações e conselhos profissionais, mas de seu reconhecimento pelo Estado (31), o que é parte da estruturação do monopólio profissional (32). No caso brasileiro essa terceira lógica ainda se encontra em posição privilegiada, o que se verifica na grande proeminência das associações profissionais no controle do acesso ao exercício da medicina, bem como no controle ético e técnico (33). A ameaça da integração pelo mercado, porém, se torna muito mais séria em decorrência dos possíveis ganhos de eficiência com a telemedicina comercial.

Essa tendência se mostra mais significativa pois, além das vantagens do modelo de negócio, o deslocamento do plano físico para o virtual é acompanhado da realocação da construção da confiança do paciente, elemento tradicionalmente ligado à confiança em profissionais específicos. A eficiência projetada pela marca e ações de propaganda e 
marketing de plataformas dominantes substituirão, ao mesmo tempo, as redes interpessoais de sustentação da reputação individual e a confiança nas instituições com suporte tradicional e burocrático $(34,35)$.

Com efeito, no caso da intermediação eletrônica de serviços é possível que a confiança se localize completamente na figura do intermediador, agindo como instância legitimadora do serviço.

Se o sistema não permite a escolha do prestador de serviço pelo consumidor, como, por exemplo, o próprio sistema da Uber, a confiança do usuário deve se centrar completamente na figura do intermediador, já que ele não possui de antemão informações sobre o prestador. Nesse caso, ao invés de confiar na autoridade pública para certificação de motoristas privados (no caso de táxis, o alvará é de competência municipal), o usuário confia particularmente na plataforma e, em um momento posterior ao ingresso na rede e de menor importância, nas avaliações de outros usuários sobre aquele provedor. Assim, haveria sobretudo o deslocamento da confiança de uma instituição para outra com um sistema de avaliação em rede que auxilia a decisão do consumidor.

Se a plataforma permite ao consumidor verificar todas as informações sobre reputação antes da decisão de aquisição do serviço, como, por exemplo, a plataforma Airbnb, a confiança se centra nas avaliações em rede, em maior medida, e na plataforma, em uma menor medida. Caso o fornecedor da plataforma não possua nenhuma avaliação anterior, a confiança do usuário naturalmente se desloca para a plataforma, que seria encarregada de realizar um escrutínio prévio para a aprovação de fornecedores. Assim, vêse que não são dois polos de um espectro de confiança, mas sim duas nuances em relação às instâncias legitimadoras da confiança do usuário.

A plataforma de telemedicina que realiza intermediação de serviços entre paciente e médico se aproxima dessas duas figuras acima retratadas (Uber e Airbnb), já que leva a um novo paradigma de confiança: a confiança na plataforma e, por consequência, na rede.

A plataforma de telemedicina não é uma instância de legitimidade do serviço médico per se, já que a prerrogativa de determinar quais profissionais são aptos a exercerem medicina deva continuar com os órgãos de classe e a sistemática pública de oferta e regulação. Tende a haver uma sobreposição de instâncias de regulação e legitimação, embora seja razoável esperar que, do ponto de vista da decisão do paciente ou do médico 
que aderem à plataforma, a marca e o tamanho do universo de prestadores ou consumidores aos que dá acesso desempenharão um papel central.

É certo, contudo, que a plataforma deve manter um mínimo de escrutínio sobre o ingresso de novos provedores de serviço para que os usuários se sintam confortáveis para confiar na rede. Entretanto, a confiança na rede é um fenômeno que se retroalimenta, tendo em vista que mais médicos e mais avaliações aumentam a robustez da rede e, por conseguinte, a sua confiabilidade do ponto de vista do consumidor. Quanto mais robusta a rede, mais interessante para médicos e pacientes dela se utilizarem, alargando a sombra do futuro e provendo o aprimoramento dos serviços médicos (36).

Dessa forma, a intermediação resolve dois problemas ínsitos à contratação de profissionais médicos: confiança e obtenção de informação (37). A plataforma pode congregar informações sobre a especialidade e histórico de desempenho, inclusive sendo capaz de sugerir profissionais adequados para as demandas específicas do usuário. Caso o indivíduo pretendesse coletar todas essas informações por conta própria, seria necessário acessar o histórico do conselho regional, verificar os dados da formação e titulação do médico, consultar as unidades hospitalares que ele trabalho e se comunicar com os seus colegas e ex-pacientes para determinar o grau de confiabilidade do profissional com a mesma precisão que a plataforma hipoteticamente pode fazer com apenas um clique. Assim, o custo de obtenção de informação se reduz, com efeitos positivos, em termos de qualidade, em todo o sistema.

\section{Médicos como usuários dos serviços das plataformas de telemedicina: profissionais, trabalhadores ou consumidores?}

A posição do médico em relação à plataforma pode ser, obviamente, matizada em razão da especialidade que exerce e da estratificação do universo dos pacientes. No Brasil é possível identificar consideráveis iniquidades no acesso à saúde, inclusive conforme o atendimento pelo SUS, por sistemas de medicina complementar e por medicina estritamente privada (38). Sem mudanças profundas na regulação e estruturação das políticas públicas é pouco provável haver uma reversão radical dessa situação exclusivamente em razão da adoção generalizada da telemedicina. A análise proposta, portanto, não tem intenção de cobrir as condições próprias da integralidade da oferta de serviços de saúde, embora possa servir como modelo de tipo-ideal para identificar dinâmicas reais. 
Considerando os tipos legais existentes no Brasil, a relação do médico com a plataforma pode ser coberta pela definição de empregado, bem como pela de consumidor. A primeira, indicada pelo art. $3^{\circ}$ da CLT (39), abrange a prestação por pessoa física, em caráter não eventual, com subordinação e recebimento de salário. O entendimento jurídico, porém, flexibiliza essas noções. É corriqueira, por exemplo, a desconsideração da franquia como um vínculo contratual comercial e sua reclassificação como relação trabalhista, desde que identificada a subordinação, da qual a cláusula de não concorrência pode ser um importante indicador (40-42).

Por outro lado, consumidores são definidos pelo art. $2^{\circ}$ do CDC (43) de maneira muito ampla: "toda pessoa física ou jurídica que adquire ou utiliza produto ou serviço como destinatário final" sem prejuízo, para fins de defesa de interesses coletivos, da equiparação de coletividades indetermináveis, o que é reforçado pelo art. 29, que inclui todas as pessoas, determináveis ou não, desde que expostas a práticas comerciais. Médicos que utilizem plataformas de intermediação são adquirentes de um serviço, do qual evidentemente são usuários finais e a própria categoria profissional dos médicos pode figurar como figura equiparada para fins de proteção coletiva de seus interesses. Portanto, é perfeitamente possível que um médico possa estar coberto por ambas as definições.

Porém, como a própria experiência da Uber vem demonstrando, as plataformas tenderão a evitar a qualificação jurídica como trabalhador, dado o interesse em evitar a proteção legal, particularmente a concessão de direitos como descanso remunerado, férias, fundo de garantia por tempo de serviço, proteção contra a demissão sem justa causa e outras figuras protetivas. Embora o Direito brasileiro venha flexibilizando os direitos dos trabalhadores, a caracterização como tal tem consequências significativas sobre os eventuais empregadores.

Em termos estritamente jurídicos, espera-se a articulação teórica e técnica de discursos e narrativas voltados a utilizar os conceitos de profissão liberal e consumidor como excludentes da categoria trabalhador. A primeira tentativa não deverá prosperar, até porque o exercício da medicina em condições de trabalho assalariado já está muito bem integrado à realidade dos tribunais e dos trabalhadores. A segunda, porém, tende a ser mais longa e complexa.

Há, nesse sentido, duas questões técnicas relevantes: i) é possível acumular as condições de trabalhador e consumidor, ou a primeira absorve a segunda? e (bi) se as 
categorias não são cumulativas, a caracterização de consumidor afasta a de trabalhador? A discussão dogmática não cabe no âmbito deste artigo, voltado a analisar as possibilidades jurídicas adequadas o cenário econômico e profissional. Cabe, porém, ressaltar não ser possível prever com tranquilidade qual será a resposta dos tribunais.

Tendo em vista que usualmente a uberização dos diversos serviços e áreas é vista somente em relação aos seus impactos trabalhistas, ela revela uma profunda diminuição na liberdade de contratar dos profissionais aderidos. A redução da importância da autonomia da vontade na celebração dos negócios jurídicos é fenômeno que vem acompanhando a evolução dos contratos no século $X X$, mas que, aparentemente, tomou ares de regra de contratação nas plataformas digitais. Esta redução da liberdade pode ocasionar efeitos e incentivos perversos na execução dos contratos. Por exemplo, o médico pode decidir seguir uma orientação da plataforma e realizar condutas médicas questionáveis, caso elas sejam conflitantes, sob o receio de ser excluído da plataforma. Outro exemplo ocorre quando o médico pede exames que não são completamente necessários, mas que de alguma forma beneficiariam o seu rating ou ranking na plataforma. Essas questões éticas também não são novas, mas prometem se intensificar diante do aumento exponencial do atendimento que grandes plataformas de intermediação são capazes de gerar. Embora cause espécie imaginar o médico violando deveres éticos e morais da sua classe para privilegiar uma plataforma digital, é razoável supor que essas plataformas podem ser desenhadas a prover fortes incentivos neste sentido.

\section{Considerações finais}

A precarização das relações de trabalho caracterizada pela uberização dos serviços acompanha uma precarização dos vínculos e da liberdade contratual dos agentes envolvidos nessas plataformas digitais. Se esste fenômeno já vinha ocorrendo paulatinamente a partir do final do xéculo XX, a sua dimensão tende a se potencializar com as novas tecnologias de informação que vêm se desenvolvendo.

A uberização da medicina revela também que não há barreira peculiar econômica ou moral, mas apenas jurídica, para a ocorrência desse fenômeno em outras áreas, como, por exemplo, a teleadvocacia, teleengenhari”, respeitados os predicados éticos dessas áreas e profissionais. 
Essas alterações também acarretam uma paulatina transferência de confiança das instituições históricas de legitimidade, como o centro hospitalar, o órgão de classe, entre outros, para a forma de confiança em rede, sobrelevando a importância econômica e social dos intermediadores. Se é aumentada a magnitude dos intermediadores, isso usualmente ocorre às custas da diminuição da figura do prestador e do usuário da plataforma.

É certa a possibilidade de categorizar os médicos que utilizem tais plataformas como consumidores, nos termos do Direito brasileiro. Mais do que isso, a categoria e as suas associações têm espaço para atuar na defesa de interesses difusos e coletivos garantidos pela legislação consumerista.

Conforme o desenvolvimento do modelo, porém, a condição de trabalhador também pode ser estendida a médicos dedicados às plataformas. A reclassificação de muitas franquias e a existência de modelos capazes de caracterizar a subordinação são indicadores dessa possiblidade.

É bastante provável que as plataformas digitais de intermediação venham a se tornar um importante agente no campo da saúde, inclusive com estratégias muito ativas de influência política sobre os processos regulatórios. Nesse sentido, a atenção dos atores públicos e da classe médica pode ser de vital importância para evitar possíveis efeitos deletérios desse novo modelo, dando amplo campo para seus efeitos positivos, inclusive na forma comercial.

\section{Referências}

1. Costa JAF, Garcia MAF. Telemedicina: o papel dos provedores de telemedicina e os vínculos jurídicos com os profissionais da saúde. Migalhas [Internet]. 2020 Ago 10 [Acesso em 20.ago.2020]. Disponível em https://www.migalhas.com.br/coluna/migalhascontratuais/331811/telemedicina-o-papel-dos-provedores-de-telemedicina-e-os-vinculosjuridicos-com-os-profissionais-da-saude.

2. Brasil. Lei no 13.989, de 15 de abril de 2020 [Acesso em 23.ago.2020]. Disponível em: http://www.planalto.gov.br/ccivil_03/_ato2019-2022/2020/Lei/L13989.htm.

3. Hafferty FW, Light DW. Professional dynamics and the changing nature of medical work. Journal of Health and Social Behavior, 1995; extra issue: 132-152.

4. Organização Mundial da Saúde. Telemedicine: opportunities and developments in Member States: report on the second global survey on eHealth. Genebra: OMS; 2010.

5. Acquier A. Uberization meets organizational theory: platform capitalism and the rebirth of the putting-out system. Academy of Management Global Proceedings, Vol. Surrey 
[Internet]. 2018 Jun 15 [Acesso em 20.abr.2020]. Disponível em

https://www.researchgate.net/publication/325320286_Uberization_meets_Organizational_T heory_Platform_capitalism_and_the_rebirth_of_the_putting-out_system

6. Pasquier T. Le droit social confronté aus défis de l'ubérisation. Dalloz IP/IT. 2017; 7-8: 368-374.

7. Nurvala JP. 'Uberisation' is the future of the digitalised labour market. European View. 2015; 14: 231-239.

8. Sharan A, Bryant L. Uberisation of housing markets: putting theory into practice. Property Management. 2017; 35(2): 202-216.

9. Young SD. Work of art in the age of technological disruption: the future of work in the music industry. MEIEA Journal [Internet]. 2018 [Acesso em 18.abr.2020]; 18(1). Disponível em https://go.gale.com/ps/i.do?id=GALE\%7CA570057921\&v=2.

$1 \& u=$ capes\&it=r\&p=AONE\&sw=w.

10. Thébaut JF. Nouvelles technologies en santé : ubérisation ou augmentation? Presse Med. 2017; 46: 561-564.

11. Williamson OE. The economic institutions of capitalism. Nova lorque: Macmillan, 1985.

12. Williamson OE. The nature of the firm: origins, evolution, and development. Oxford: OUP, 1991.

13. Eggertsson T. Economic behaviour and institutions principles of neoinstitutional economics. Cambridge: CUP, 1990.

14. Assembleia Médica Mundial. Statement on Accountability, Responsibilities and Ethical Guidelines in the Practice of Telemedicine [Internet]. Out. 1999 [Acesso em 3.abr.2020]. Disponível em: https://www.wma.net/policies-post/wma-statement-on-accountabilityresponsibilities-and-ethical-guidelines-in-the-practice-of-telemedicine/.

15. Maldonado J, Marques A, Cruz A. Telemedicina: desafios à sua difusão no Brasil. Cadernos de Saúde Pública. 2016; 32(2): 1-12.

16. Luxton D. Considerations for Planning and Evaluating Economic Analyses of Telemental Health. Psychological Services. 2013; 10(3): 276-282.

17. Torre-Diez I, López-Coronado M, Vaca C, Aguado JS, Castro C. Cost-utility and costefectiveness studies of telemedicine, electronic, and mobile health systems in the literature: a systematic review. Telemedicine Journal and E-Health. 2015 fev; 21(2): 81-85.

18. Freed J, Lowe C, Flodgren G, Binks R, Doughty K, Kolsi J. Telemedicine: is it really worth it? A perspective from evidence and experience. Journal of Innovation in Health Informatics. 2018; 25(1): 14-18. 
19. Kahn J, Rak K, Kuza C, Aschcraft L, Barnato A, Fleck J et al. Determinants of Intensive Care Unit Telemedicine Effectiveness. An Ethnographic Study. 2019 abr; 199(8): 970-979.

20. Hall M, Schneider CE. Patients as consumers: courts, contracts, and the new medical marketplace. Michigan Law Review. 2007/2008 106: 643-689.

21. Macdonald KM. The sociology of professions. Nova lorque: Sage;1995.

22. Freidson E. Profession of medicine: a study of the sociology of applied knowledge. Nova lorque: Dood, Mead \& Company; 1970.

23. Foucault M. El nacimiento de la clínica: una arqueología de la mirada médica. Buenos Aires: Siglo XXI; 2012.

24. Larson M. Professionalism: the third logic (review). Perspectives in Biology and Medicine. 2003 46(3): 458-462.

25. Freidson E. Professionalism, the third logic: on the practice of knowledge. Chicago: University of Chicago Press; 2001.

26. Mckinlay JB, Arches J. Towards the proletarization of physicians. International Journal of Health Services. 1985; 15(2): 161-195.

27. Coburn D. Professionalization and proletarization: medicine, nursing, and chiropractic in historical perspective. Labour/Le Travail. 1994; 34(Fall): 139-162.

28. Dent M. Professionalism, educated labour and the state: hospital, medicine, and the new managerialism. The Sociological Review. 1993; 41(2): 244-273.

29. Santos PM. Profissão médica no Brasil. Machado MH (Org.) Profissões da Saúde: uma abordagem sociológica [Internet]. Rio de Janeiro: Fiocruz; 1995 [Acesso em 10.fev.2020]. p. 97-117. Disponível em http://books.scielo.org/id/t4ksj/epub/machado9788575416075.epub.

30. Southon G, Braithwaite J. The end of professionalism? Social Science and Medicine. 1998; 46(1): 23-28.

31. Abbott A. The system of professions: an essay on the division of expert labor. Chicago: University of Chicago Press; 1988.

32. Larson MS. The rise of professionalism: a sociological analysis. Berkeley, University of California; 1977.

33. Castro JV, Balbinot RA. Panorama jurídico institucional das especialidades médicas no Brasil. Aith F, Balbinot RA, Ventura D, Dallari SG (Orgs.) Regulação de profissões de saúde no Brasil e em perspectiva comparada. São Paulo: CEPEDISA; 2020: 57-79. 
34. Erp J. Reputational Sanctions in Private and Public Regulation. Erasmus Law Review. 2009 1(5): 145-162.

35. Botsman R. Who can you Trust? How Technology Brought Us Together and Why It Might Drive Us Apart. Nova lorque: Penguim Books; 2017.

36. Axelrod R. The Evolution of Cooperation. Nova lorque: Basic Books; 1984.

37. Milgron $P$, North $D$, Weingast $B$. The Role of Institutions in the Revival of Trade: the Law Merchant, Private Judges and the Champagne Fairs. Economics and Politics. 1990 mar; 2(1): 1-23.

38. Travassos C, Castro MSM. Determinantes e desigualdades sociais no acesso e na utilização de serviços de saúde. Giovanella L, Escorel S, Lobato LVC, Noronha JC, Carvalho Al (orgs). Rio de Janeiro: Fiocruz; 2012: 183-206.

39. Brasil. Consolidação das Leis do Trabalho, maio de 1943 [Acesso em 1.jul.2020]. Disponível em: http://www.planalto.gov.br/ccivil_03/decreto-lei/del5452.htm.

40. Almeida APKO, Almeida Júnior JGC. Contrato de franquia: possíveis problemas da relação contratual. Revista de Direito Privado. 2018; 94: 99-115.

41. Pimenta JRF, Porto LV, Rocha CJ. A dependência econômica como critério identificador da relação de emprego. Revista de Direito do Trabalho. 2019 out; 206: 19-44.

42. Martins SP. O franchising como forma de terceirização. Revista de Direito do Trabalho. 1996; 95: 33-42.

43. Brasil. Código de Defesa do Consumidor. Brasília, setembro de 1990 [Acesso em 1.jul.2020]. Disponível em: http://www.planalto.gov.br/ccivil_03/leis/l8078.htm.

\section{Colaboradores}

Costa JAF contribuiu com a concepção, desenho e redação final do artigo. Sola $F$ contribuiu com a redação e levantamento e análise dos dados do artigo. Garcia MAF contribuiu com a redação e levantamento e análise dos dados do artigo. 